\title{
Rewiring of the cellular and inter-cellular landscape of the human colon during ulcerative colitis
}

Christopher S. Smillie, Moshe Biton, Jose Ordovas-Montanes, Keri M. Sullivan, Grace Burgin, Daniel B. Graham, Rebecca H. Herbst, Noga Rogel, Michal Slyper, Julia Waldman, Malika Sud, Elizabeth Andrews, Adam L. Haber, Sanja Vickovic, Danielle Dionne, Lan T. Nguyen, Alexandra Chloé Villani, Matan Hofree, Elizabeth A. Creasey, Hailiang Huang, Orit Rozenblatt-Rosen, John J. Garber, Hamed Khalili, A. Nicole Desch, Mark J. Daly, Ashwin N. Ananthakrishnan, Alex K. Shalek, Ramnik J. Xavier, and Aviv Regev

The paper has been withdrawn owing to erroneous inclusion of confidential information relating to a third party. 